\title{
Computer simulation and visualization of supersonic jet for gas cluster equipment
}

\author{
A. Ieshkin ${ }^{\text {a }}$, Y. Ermakov ${ }^{\text {b }}$, V. Chernysh ${ }^{\text {a }}$, I. Ivanov ${ }^{\text {a }}$, I. Kryukov ${ }^{c}$, K. Alekseev $^{\text {d }}$, N. Kargin ${ }^{\text {d }}$, \\ Z. Insepov ${ }^{\mathrm{e}, \mathrm{f}, *}$ \\ ${ }^{a}$ Faculty of Physics, Lomonosov Moscow State University, Leninskie Gory, Moscow 119991, Russia \\ b Scobeltsyn Nuclear Physics Research Institute, Lomonosov State Moscow University, GSP-1, Leninskiye Gory, Moscow 119991, Russia \\ ' Institute for Problems in Mechanics, Russian Academy of Sciences, prosp. Vernadskogo, 101, Block 1, Moscow 119526, Russia \\ ${ }^{\mathrm{d}}$ National Research Nuclear University "MEPhI», Kashirskoye shosse 31, Moscow 115409, Russia \\ e Purdue University, 500 Central Drive, West Lafayette, IN, USA \\ ${ }^{\mathrm{f}}$ Nazarbayev University Research and Innovation System, Kabanbay Batyr Avenue 53, Astana, Kazakhstan
}

\section{A R T I C L E I N F O}

Article history:

Received 30 March 2015

Received in revised form

1 June 2015

Accepted 13 June 2015

Available online 20 June 2015

Keywords:

Cluster ions

Flow visualization

Supersonic nozzle

\begin{abstract}
A B S T R A C T
Supersonic nozzle is a key component of a gas cluster condensation system. We describe a flow visualization system using glow discharge with annular or plane electrodes. The geometric parameters of a supersonic jet under typical conditions used in a gas cluster ion beam accelerator are investigated. As well numerical simulations were performed. Dependence of inlet and ambient pressures and nozzle throat diameter on the shock bottle dimensions is described for different working gases. Influence of condensation rate on shock bottle axial size is discussed.
\end{abstract}

(c) 2015 Elsevier B.V. All rights reserved.

\section{Introduction}

Recent decades, gas cluster ion beams (GCIB) have been under extensive study. GCIB are widely used in practical applications, such as precise surface polishing and etching, ultra-shallow implantation, ion-assisted deposition of thin films, probing in SIMS technique [1-3], as well as in investigations of fundamental properties of matter $[4,5]$.

The usual way of obtaining gas clusters is adiabatic expansion of a working gas through a supersonic nozzle, so that expanded gas becomes cold enough for clusterization. Information on properties of the jet below a nozzle is essential for optimization a nozzle and skimmer geometry and the distance between them.

The typical structure of a supersonic jet shown in Fig. 1 is described in [6,7]. It is shown in Fig. 1, that the gas expanding to the ambient pressure $p_{\infty}$ forms a "shock bottle" configuration. Expansion waves reflect from the jet boundary as weak compression waves and form an oblique shock. Behind the oblique shock,

\footnotetext{
* Corresponding author at: Purdue University, 500 Central Drive, West Lafayette, IN, USA.

E-mail address: zinsepov@purdue.edu (Z. Insepov).
}

the flow is supersonic as well, but its Mach number is less than in the jet core.

In the core, the flow is isentropic, and ideal gas equations are correct for it. In the low-temperature media of the expanding gas condensation starts and clusters can exist. Then, passing through the normal shock, which is called Mach disc, entropy increases: the gas gets warm and clusters are likely to dissociate. To prevent this dissociation, a skimmer is used. It cuts the Mach disk, penetrates into the core of the jet and evacuates clusters before they collapse. The skimmer should be distant enough from the nozzle in order to let the clusters grow. On the other hand, it should not disturb the shock bottle structure. So, knowing the form and the structure of the flow is essential for optimizing cluster ion sources.

The picture given attributes to a jet from a sonic nozzle, i.e. a nozzle with only a converging part. However, a supersonic nozzle consisting of converging and diverging parts is usually used to generate GCIB [1,3]. Unfortunately, for such a nozzle we could not find any information on a jet structure under extensive clusterization. Typical ways of observing gas flows are rather complicated techniques such as schlieren-photography, electron-excited luminescence or Raman scattering [6-8]. Besides, these techniques demand additional equipment and substantial changes of the 
cluster formation chamber of a GCIB accelerator. Recently suggested technique utilizing dielectric barrier discharge [9] was not able to reveal the structure of the jet [10].

In this work, we suggest a simple technique of visualization of a jet by using glow discharge. It is suitable for using under pressure rates existing in GCIB accelerators, has very low requirements on the equipment and makes it possible to see the jet structure with a naked eye. Results of both experimental investigations and computer simulations of a supersonic jet are presented.

\section{Experiment}

The experiments were carried out using GCIB accelerator of Moscow State University [3]. The accelerator operates in pulsed mode. It means that the working gas is supplied through a pulsed valve installed before the nozzle. The pulse length was selected to be long enough for taking a photo.

The upper cover of the cluster formation chamber was made transparent. The skimmer was removed to provide more space for the experiments. Two configurations of electrodes were used to initiate glow discharge: an annular electrode and plane electrodes. Both configurations are shown in Fig. 2.

The annular electrode made of tungsten wire was placed coaxially with the nozzle. It had a diameter of $54 \mathrm{~mm}$. The ring was under DC voltage, while the nozzle was grounded as well as the chamber walls and acted as the second electrode.

The two plane copper electrodes could be used instead of the annular one. They were placed symmetrically with respect to the nozzle axis. The distance between them was $65 \mathrm{~mm}$; the longitudinal dimension was $62 \mathrm{~mm}$. One of the electrodes was under

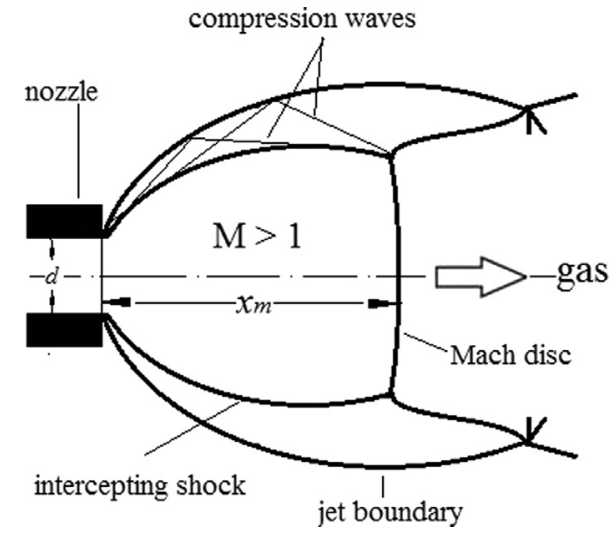

Fig. 1. Schematic view of a gas jet from a sonic nozzle.
DC voltage; the other was grounded as well as the nozzle and the chamber walls.

In both cases, nozzle position relatively to the electrodes was set by motion of the nozzle holder.

To study the geometrical parameters of the jet depending on the inlet pressure and ambient pressure we used conical nozzles with critical cross-section diameters $140 \mu \mathrm{m}$ and $400 \mu \mathrm{m}$. The length of diverging part and the half-opening angle were $20 \mathrm{~mm}$ and $6^{\circ}$, respectively.

Maximum pressure in the chamber was limited by the capability of the pumping system. In the operating mode of the accelerator, it does not exceed $8 \cdot 10^{-3}$ Torr. Depending on the duty factor of the pulsed valve and type of the working gas it can be raised up to $5 \cdot 10^{-2}$ Torr for a short time. Under such pressures, the glow discharge is initiated by the voltage of $0.3-2.5 \mathrm{kV}$ depending on the experimental conditions.

For particular experimental conditions (working gas type and pressure, duty factor of the pulsed valve, configuration and position of the nozzle and electrodes) optimal values of the voltage and current exist to make a discharge snapshot. For example, excessive discharge current results in overexposed snapshot, and the jet structure is hardly distinguished. At the same time, insufficient current leads to low light intensity of the discharge.

Along with the experiments, computer simulations of a viscous gas flow in the axisymmetric nozzle and jet were performed. The flow was modeled by the system of two-dimensional unsteady Navier-Stokes-Fourier (NSF) equations. The system was written out in the divergence form and supplemented by the equations of state of an ideal gas and the boundary conditions on the boundaries of the computational domain. Terms describing convective transport in this system were approximated using a modified Godunov's scheme of high order of accuracy [11]. Terms describing diffusive (viscous) transfer were approximated using the finite volume method, which in the case of a uniform grid is reduced to a central difference approximation of derivatives. Advance in time was performed using the third order Runge-Kutta method. Influence of the gas condensation on the stream parameters was not taken into account on this stage.

Since the expansion ratio in the nozzle and jet is high (more than 1000), and at inlet temperature, the temperature at the nozzle exit falls below the critical value. Therefore temperature dependence of dynamic viscosity $\mu$ is described with the modified Satherland expression [12]:

$$
\begin{aligned}
\mu= & \left\{\mu\left(T_{*}\right)\left(T / T_{*}\right)^{a}, T<T_{*}\right. \\
& \mu\left(T_{*}\right)\left(T / T_{*}\right)^{3 / 2}\left(T_{*}+S\right) /(T+S), T \geq T_{*}
\end{aligned}
$$

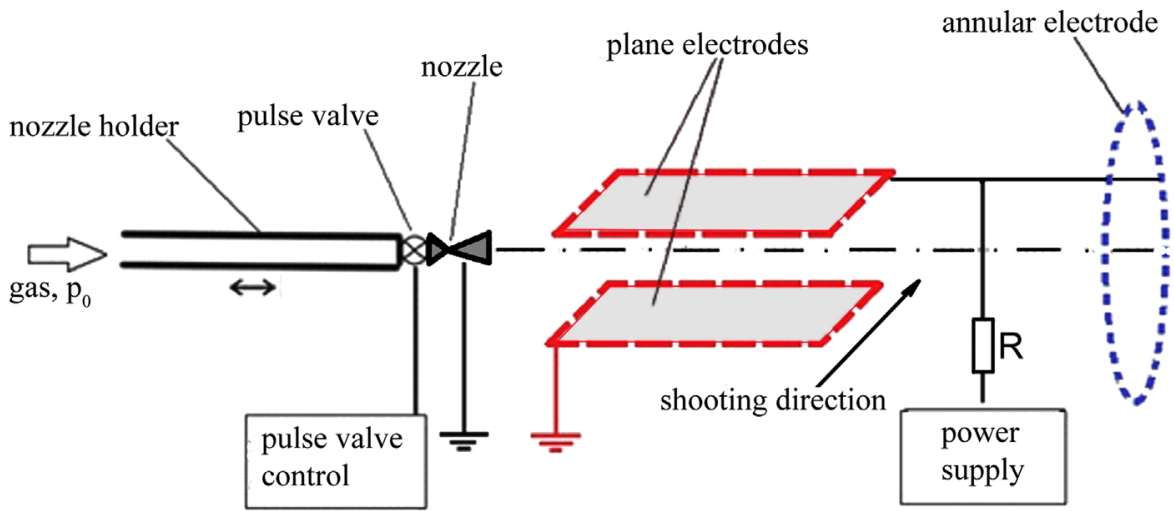

Fig. 2. Schematic diagram of flow visualization system using plane electrodes or an annular electrode. 
where reference temperature $T_{*}$ is the critical temperature ( $T_{*}=150 \mathrm{~K}$ for argon), $S=128.35 \mathrm{~K}, a=0.945$.

Possible effects of rarefaction near the solid surfaces were considered with slip boundary conditions and first order temperature jump near the wall [13]:

$$
\begin{aligned}
& u_{\text {slip }}=u_{s}-u_{\text {wall }}=\frac{2-\sigma}{\sigma} \lambda \frac{\partial u_{s}}{\partial n}+\frac{3}{4} \frac{\mu}{\rho T_{G}} \frac{\partial T_{\text {wall }}}{\partial s} \\
& T_{\text {jump }}=T_{G}-T_{\text {wall }}=\frac{2-\sigma_{T}}{\sigma_{T}} \frac{2 \gamma}{\gamma+1} \frac{\lambda}{\operatorname{Pr}} \frac{\partial T_{G}}{\partial n}
\end{aligned}
$$

where $\sigma$ - accommodation coefficient of the tangential momentum; $\sigma_{T}$ - accommodation coefficient of temperature, $\sigma_{T}=1$ under diffuse scattering of the molecules by the wall and $\sigma_{T}=0$ under specular reflection; $n$ and $s$ are the normal and tangential to the wall vectors; $u_{s}$ - gas velocity along the wall, $u_{\text {wall }}$ - wall velocity in the direction of the tangential vector; $T_{G}$ - gas temperature, $T_{\text {wall }}-$ wall temperature (in our case, $T_{\text {wall }}=297 \mathrm{~K}=$ constant); $\lambda$ - mean free path of the atoms, $\lambda=16 \mu /\left(5 \rho \sqrt{2 \pi R T_{G}}\right) ; \rho$ - gas density; $R-$ gas constant; $\gamma$ - ratio of heat capacities; $\operatorname{Pr}$ - Prandtl number.

As the result of the simulations maps of density, temperature, pressure and Mach numbers could be obtained.

\section{Results and discussion}

\subsection{Annular electrode}

The main advantage of the annular electrode system is its axial symmetry. It means that the distribution of luminescence in the radial direction is not distorted by the discharge irregularities, such as dark spaces.

If the electrode was under positive voltage, the discharge appeared between the electrode and chamber walls at voltage as low as $350 \mathrm{~V}$. This meant high current and overexposed snapshot. If the electrode was under negative voltage, the discharge was initiated between the electrode and the nozzle. In this case the jet structure was revealed from the distribution of visible light from the discharge.

Fig. 3a shows the picture of discharge between the nozzle and the cathode. At large distances, the picture does not change. More intensive luminescence can be interpreted as higher density and

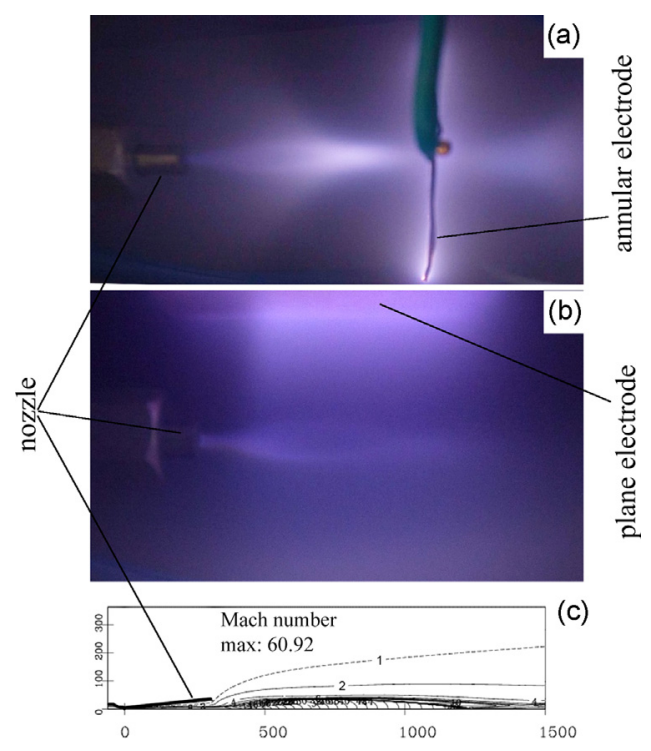

Fig. 3. Experimental argon flow visualization with glow discharge. $140 \mu \mathrm{m}$ nozzle, annular (a) and plane (b) electrodes; computer simulation of Mach number distribution in argon flow, spatial coordinates are shown in nozzle radius units (c). temperature at the jet border [14]. Less intensive luminescence means lower density and temperature in the core of the jet. The picture makes it possible to identify the geometric parameters of the shock bottle. We should note that its form differs from the one shown in Fig. 1. In particular, the normal shock has no finite diameter and approaches the axis. This is typical for rarefied streams. Downstream from the Mach barrel luminescence on the axis remains more intensive.

Decreasing the distance between the nozzle and the cathode, dark spaces rounding the wire become more important. They overlap the region of the jet luminescence, so it is hard to understand, if the glow distribution was determined by the jet itself or affected by the dark regions. The luminescence weakens near the cathode plane, but intensifies again after passing it.

When the cathode plane coincides with the normal shock position and at even less distances between the cathode and the nozzle, this decrease of intensity disappears and the jet is visualized without interruption.

Thus, flow visualization with annular electrode allows to determine the jet form and the normal shock position, but the luminescence picture can be distorted at small distances between the nozzle and the cathode.

\subsection{Plane electrodes}

Though in the case of plane electrodes the system is not axisymmetric, the shape of visible jet is the same as in case of annular electrode and it is symmetric with respect to the central plane. It means that the picture of luminescence is not distorted by the electric field. Nozzle longitudinal position with respect to the electrodes does not affect the shape of the jet as well as the value of discharge voltage and current over the ranges used.

Geometrical parameters of the experimentally obtained pictures are in good agreement with computer simulations (Fig. 3).

$\mathrm{Ne}, \mathrm{N}_{2}, \mathrm{Ar}$ and Xe were used as the working gases. For each nozzle experiments with different inlet pressures were conducted. The inlet pressure varied from 4 bar to 7 bar for the $140 \mu \mathrm{m}$ nozzle and from 1 bar to 3 bar for $400 \mu \mathrm{m}$ nozzle. The ambient pressure in the chamber changed in proportion to the inlet pressure. The pressures ratio was individual for each type of gas and was governed by its influx and pumping rate.

Because of large free path in a rarefied gas, the shock wave front is spread. In all experiments the position of the center of the front was evaluated. As well, the Mach disk position was calculated using expression obtained in [4]:

$\frac{x_{m}}{d}=0.67 \sqrt{\frac{p_{0}}{p_{\infty}}}$

where $p_{0}$ and $p_{\infty}$ are inlet and ambient pressures, respectively; $d$ and $x_{m}$ are shown in Fig. 1 .

Simulations were conducted for argon flow under inlet pressures 3 bar, 5 bar and 7 bar for $140 \mu \mathrm{m}$ nozzle and 2 bar for $400 \mu \mathrm{m}$ nozzle. As an example of calculated distributions, dependences of density and longitudinal velocity along the axis on the distance from the nozzle cross-section, are given in Fig. 4. This figure shows that the velocity rapidly increases inside the nozzle. The density distribution has two regions of rapid decrease, which correspond to expansion inside the nozzle and to free expansion. Both dependencies clearly indicate the position of the Mach disk.

The lengths of Mach barrel obtained from the experiments and simulations for different pressures and different nozzles are shown in Fig. 5. Each experimental point represents data obtained for different inlet pressures but the same inlet and ambient pressures ratio. It is found that both experimental and simulated values of the distance exceed calculated ones. 


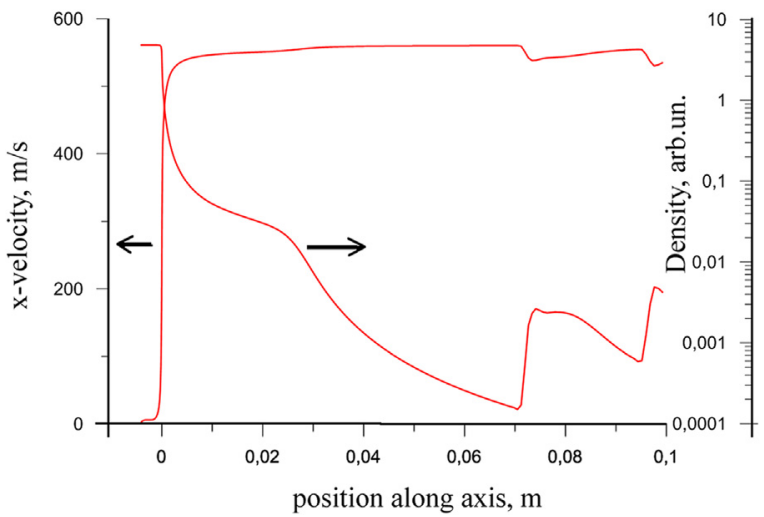

Fig. 4. Distribution of density and longitudinal velocity along the stream axis. $140 \mu \mathrm{m}$ nozzle, the distance is calculated from the nozzle critical cross-section.

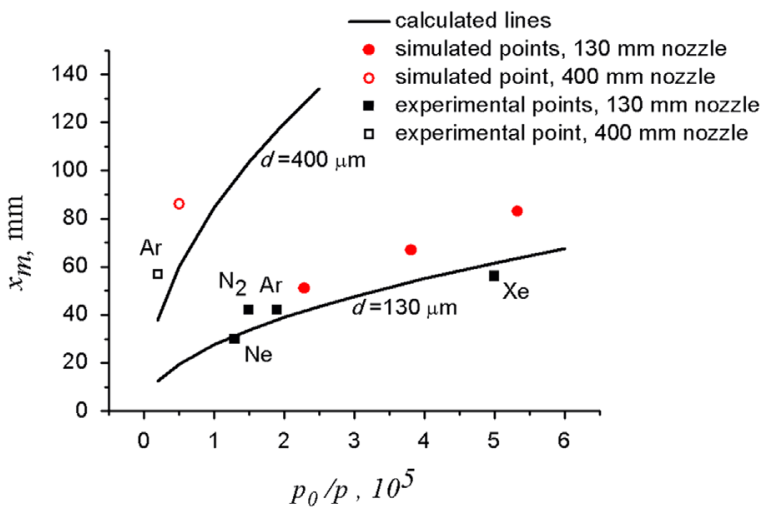

Fig. 5. Distance from sonic nozzle exit to Mach disc. Calculated using Eq. (4) (lines): results of simulations (circles) and experiments (squares) for converging-diverging nozzle of different critical cross-section.

Table 1

Axial sizes of the jet and condensation parameters for the used gases, from [15].

\begin{tabular}{lcc}
\hline Gas & Axial jet size $(\mathrm{mm})$ & Condensation parameter \\
\hline $\mathrm{Ne}$ & 5 & 185 \\
$\mathrm{~N}_{2}$ & 8 & 528 \\
$\mathrm{Ar}$ & 10 & 1650 \\
$\mathrm{Xe}$ & 16 & 5500 \\
\hline
\end{tabular}

The length of Mach barrel is affected by the walls of the diverging part of the nozzle, which limit axial expansion of the flow causing increase of the distance to Mach disk. On the other hand, Eq. (4) is obtained for converging nozzles with orifice of some millimeter. For supersonic nozzles with critical cross-section on the order of $100 \mu \mathrm{m}$, viscosity diminishes effective crosssection, causing decrease of the Mach barrel length. As it is shown in Fig. 4, positive deviation from the calculated value is larger for the $400 \mu \mathrm{m}$ nozzle, which is apparently because of reducing the viscosity influence.

The axial size of Mach barrel did not change significantly for different inlet pressures, but changed for different gases (Table 1). This can be explained considering different degrees of clusterization.
Mean size of clusters in the expanding jet is proportional to condensation parameter, which is constant, related to cluster bond formation [15], given in Table 1. The parameter is smallest for Ne and largest for Xe. More effective condensation results in pressure increase in the jet core. So, the jet bound, where the pressure is equal to the ambient pressure, shifts from the axis. The longitudinal size of the jet is not changed. For $\mathrm{N}_{2}$, condensation parameter is smaller than for Ar, but additional widening is caused by fewer ratio of heat capacities.

\section{Conclusion}

We adapted the technique of gas flow visualization using glow discharge for gas cluster ion beam equipment conditions. This technique allows evaluation of supersonic jet geometrical dimensions and optimization of the nozzle and skimmer position and shape; the jet structure can be revealed well. Both experiments and computer simulations demonstrated that the length of Mach barrel depends on the ratio of inlet and ambient pressures in the system and can be evaluated using expression obtained for a sonic nozzle. Axial size of the jet depends as well on the type of working gas and is increases with the increase of the degree of clusterization.

\section{Acknowledgments}

The work is supported by the Ministry of Education and Science of Russia, Project no. 14.578.21.0064. One of the authors (ZI) wishes to thank the Ministry of Science and Education of Kazakhstan for partial support through the World Science Stars program, under Grant no. 031-2013 of 12/3/2013.

\section{References}

[1] I. Yamada, Applied Surface Science 310 (2014) 77. http://dx.doi.org/10.1016/j. apsusc.2014.03.147.

[2] V.N. Popok, Materials Science and Engineering R 72 (2011) 137. http://dx.doi org/10.1016/j.mser.2011.03.001.

[3] A.A. Andreev, V.S. Chernysh, Yu. A. Ermakov, A.E. Ieshkin, Vaccum 91 (2013) 47. http://dx.doi.org/10.1016/j.vacuum.2012.11.001.

[4] J. Samela, K. Nordlund, J. Keinonen, V.N. Popok, E.E.B. Campbell, The European Physical Journal D 43 (2007) 181. http://dx.doi.org/10.1140/epjd/e2007-00104y.

[5] M. Benguerba, The European Physical Journal D 66 (2012) 208. http://dx.doi org/10.1140/epjd/e2012-20521-y.

[6] S. Crist, P.M. Sherman, D.R. Glass, AIAA Journal 4 (1966) 68.

[7] G. Tejeda, B. Maté, J.M. Fernández-Sánchez, S. Montero, Physical Review Letters 76 (1996) 34. http://dx.doi.org/10.1103/PhysRevLett.76.34.

[8] L.A. Gochberg, Progress in Aerospace Sciences 33 (431-459) (1997) 461. http: //dx.doi.org/10.1016/S0376-0421(97)00002-X

[9] K. Luria, N. Lavie, U. Even, The Review of Scientific Instruments 80 (2009) 104102-1http://dx.doi.org/10.1063/1.3244085.

[10] K. Luria, W. Christen, U. Even, The Journal of Physical Chemistry A 115 (2011) 7362. http://dx.doi.org/10.1021/jp201342u.

[11] G.S. Glushko, I.E. Ivanov, I.A. Kryukov, Mathematical Models and Computer Simulations 2 (2010) 407. http://dx.doi.org/10.1134/S2070048210040010.

[12] D.Y. Dubov, in: Proceedings of All-Russian Conference Contemporary Problems of Rarefied Gas Dynamics, Novosibirsk, Russia, 2013, p. 85.

[13] S. Roy, R. Raju, H.F. Chuang, B.A. Cruden, M. Meyyappan, Journal of Applied Physics 93 (2003) 4870. http://dx.doi.org/10.1063/1.1559936.

[14] W. Merzkirch, Flow Visualization, 2nd edition, Academic Press, 1987.

[15] R.A. Smith, T. Ditmire, J.W.G. Tisch, Review of Scientific Instruments 69 (1998) 3798. http://dx.doi.org/10.1063/1.1149181. 